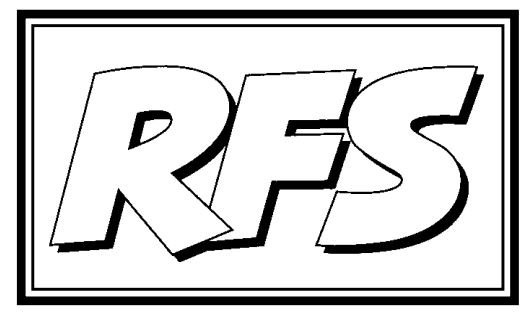

Revista de Fomento Social, 60 (2005), 39-55

\title{
Ética de las profesiones y formación universitaria: tres modelos de responsabilidad profesional $^{1}$
}

\section{Agustín DOMINGO MORATALLA ${ }^{2}$}

(PALABRAS CLAVE: ÉtICA, RESPONSABILIDAD PROFESIONAL, FoRMACIÓN UNIVERSITARIA, VALORES, CAPITAL HUMANO, CAPITAL SOCIAL, IDENTIDAD PROFESIONAL)

(KEY WORDS: Ethics, PROFESSIONAL RESPONSIBILITY, UNIVERSITY EDUCATION, VALUES, HuMAN CAPITAL, SOCIAL CAPITAL, PROFESSIONAL IDENTITY)

1 Este trabajo forma parte del Proyecto de investigación científica y desarrollo tecnológico HUM2004-06633-C02-01/FISO, financiado por el MEC y los fondos FEDER, en el marco del grupo de I+D+I 03/179 de la Generalidad Valenciana.

2 Profesor titular de Filosofía del Derecho, Moral y Política, Universidad de Valencia. 
La universidad no ofrece actualmente ningún rostro característico al joven. Éste encuentra una democracia de las disciplinas que estân allí, o bien porque son autóctonas, o bien porque han penetrado allí recientemente para cumplir alguna tarea que se esperaba de la universidad. Esta democracia es, en realidad, una anarquía, porque no hay normas reconocidas para la ciudadanía ni títulos legítimos para gobernar. No hay, en suma, ninguna visión, ni un conjunto de visiones contrapuestas, de qué es un ser humano educado. La cuestión ha desaparecido, pues plantearla supondría una amenaza para la paz. No existe ninguna organización de las ciencias, ningún árbol del conocimiento. ${ }^{3}$

\section{Introducción}

Esta imagen desconcertante de la vida universitaria es más habitual de lo que nos imaginamos. Cada día es más difícil encontrar en el mundo universitario una visión o un conjunto de visiones, aunque fueran contrapuestas, sobre lo que Bloom llama "un ser humano educado". Se trata de una pregunta inquietante cuya respuesta es más compleja de lo que se imaginan quienes consideran que la modernidad, el humanismo o los humanismos en sus múltiples versiones pueden ser la respuesta más adecuada. Una respuesta para la que no estamos sobrados de reflexiones y en la que cada día vemos con más claridad que tiene que pasar por una meditación sobre la vocación y la responsabilidad profesional como ya hiciera M. Weber o por la "misión" de la universidad a la que se refería Ortega. ${ }^{4}$

El objetivo de este artículo es promover la reflexión sobre el modelo de responsabilidad profesional con el que estamos organizando el quehacer universitario. A excepción de titulaciones donde parece clara la necesidad de una ética profesional (Medicina, Enfermería, Periodismo), en las universidades sólo con dificultad o excepcionalmente se plantea de manera explícita el lugar que un área o materia como Ética de las profesiones puede tener en la formación de alumnos, profesores y todo el personal universitario. A medida que los profesores asumen responsabilidades en la dirección, gestión, y administración de los centros se ven obligados a implicarse en programas de políticas públicas que antes o después les exigen plantearse el valor hu-

3 A. Bloom, (1989), El cierre de la mente moderna, Barcelona, Plaza y Janés, p. 348.

4 Sobre las relaciones entre la ética contemporánea de la responsabilidad y la institución universitaria puede verse mi estudio "El intelectual y la universidad. La reanimación intelectual de la vida universitaria”, en F. Torralba y J. M. Esquirol (eds.), (2000), Perplejidades y paradojas de la vida universitaria, Madrid, Caparrós, pp. 115-135. 
mano, sentido profesional y la aplicación funcional del conocimiento en la sociedad de la información.

El tema es más grave cuando los expertos en los programas de calidad ${ }^{5}$ plantean a los servicios o áreas de gestión universitaria qué indicadores disponen para evaluar la misión o visión, la asignación de procesos, la determinación de los grupos de interés, la responsabilidad social de la organización, su compromiso con la sociedad o lo que en contextos europeos llamamos Responsabilidad Social Corporativa. Las respuestas suelen ser poco comprometedoras y se suelen realizar acudiendo a la exposición de motivos de la legislación que posibilitó la creación de la universidad, al ideario con el que se trabaja si se trata de un centro privado o acudiendo a la cosmovisión política de quien ejerce en ese momento una responsabilidad.

Aunque la creación de un área o materia que lleve el nombre de Ética de las profesiones no soluciona este problema, su planteamiento puede contribuir a que los diferentes estamentos universitarios evalúen el uso que hacen de los recursos y, sobre todo, se pregunten por el perfil del profesional que están capacitando. Este hecho es especialmente importante cuando las diferentes universidades europeas han iniciado un proceso de convergencia por el que tendrán que coordinar programas, titulaciones y áreas de conocimiento. $\mathrm{Si}$ en lugar de defender únicamente los intereses propios de cada área, centro o titulación comenzásemos a preguntarnos por el perfil humano de los profesionales que estamos capacitando, probablemente perderíamos menos tiempo en unas reformas instrumentales.

No pretendo promover una reflexión corporativista que incentive y promueva un mayor número de "créditos" para quienes imparten docencia en Ética General o Ética de las Profesiones. Pretendo abrir un debate sobre el servicio que la universidad presta a la sociedad y promover una cultura de la responsabilidad en unas comunidades universitarias donde el principio de autonomía universitaria parece ser un recurso que inmuniza para evaluar con responsabilidad los recursos, los procesos, los resultados y la cultura organizativa que la institución ha puesto en marcha en la misión de capacitar profesionalmente a los estudiantes.

Quiero centrar este debate en el tema de la responsabilidad profesional y en tres posibles enfoques de la misma, a partir de un planteamiento general y sin entrar en cada uno de los perfiles exigibles en las diferentes

5 Sobre todo en modelos ISO y EFQM. 
áreas de conocimiento. Para ello, he organizado la reflexión en cuatro partes. Primero explico lo que voy a llamar vocación mediadora de la Ética de las profesiones, después enumero algunas razones por las que considero necesario introducir este debate en la vida universitaria y me centro en la que considero más importante: la posibilidad de tender un puente entre lo que técnicamente llamamos capital humano y capital social. Desarrollo esta reflexión sirviéndome del potencial ético de la hermenéutica filosófica y el personalismo comunitario. ${ }^{6}$

En la última parte presento tres modelos de responsabilidad profesional completando una ética social que ya he aplicado a otros ámbitos de la vida comunitaria como el de las organizaciones del voluntariado y las organizaciones educativas ${ }^{7}$. Aunque los tres modelos precisan de un desarrollo más detallado me limito a presentarlos de la forma más sencilla posible para que puedan ser debatidos, discutidos y utilizados en grupos de estudiantes, profesores o directivos universitarios interesados en plantearse con seriedad y radicalidad la relación que hay entre el discurso institucional (idearios) y las exigencias sociales (valores y perfiles profesionales).

\section{La vocación mediadora de la Ética de las profesiones ${ }^{8}$}

Es habitual entender por "ética de las profesiones" una Ética General que prepara para el ejercicio especializado de las profesiones para las que, a priori, capacita teóricamente la formación universitaria. Como ética general prepara el campo de forma propedéutica para los problemas que se abordarán en cada una de las éticas aplicadas correspondientes, o como viene siendo habitual en algunos centros, las correspondientes "deonto-

6 Sobre la hermenéutica filosófica puede verse nuestro trabajo, (1991), El arte de poder no tener razón. La hermenéutica dialógica de H. G. Gadamer, Salamanca, Universidad Pontificia de Salamanca; y sobre el personalismo comunitario, véanse nuestros trabajos, (2001), Un humanismo del siglo XX: El personalismo (sexta edición), Pedagógicas, e "Introducción, traducción y notas" a P. RicoeuR, (1999), Lo justo. I, Madrid, Caparrós.

7 Cf. mis trabajos, (1998), Ética y voluntariado. Una solidaridad sin fronteras (segunda edición), Madrid, PPC, y, (2002), Calidad Educativa y Justicia social, Madrid, PPC.

8 Una parte de este trabajo incorpora la intervención realizada el día 23 de Abril de 2004, en el Foro Internacional Ética y Educación, organizado por UNESCO-IFIE en la ciudad de México. Algunas ideas, que allí presenté y ahora reformulo, fueron recogidas en "La ética de las profesiones en la formación universitaria: ¿nihilismo o responsabilidad?”, (2004), Didac 43, pp. 27-35. 
logías profesionales", entendidas éstas en un sentido amplio, es decir, no reducidas al conjunto de "deberes", imperativos o normas que prescriben unas prácticas profesionales. Así entendida, una tarea urgente para la ética de las profesiones es la recuperación de su vocación mediadora. Mediar no sólo para resolver conflictos o articular la necesaria interdisciplinariedad en la gestión del conocimiento, sino para tender el puente cada vez más necesario entre las instituciones educativas y el fortalecimiento de valores como la justicia o la solidaridad. ${ }^{9}$

Se trata de mediar para generar espacios de diálogo social y, sobre todo, mediar para despertar la conciencia crítica de las instituciones que gestionan el conocimiento. Así planteadas, las éticas profesionales son un antídoto contra la tendencia al autismo de las áreas, los departamentos y las áreas del conocimiento en los que se organizan las universidades. Un antídoto que también actúa contra la tendencia a la abstracción que se produce cuando los propios profesionales se refugian en sus particulares intereses. También un antídoto para evitar el blindaje corporativo del egoísta ilustrado que limita sus responsabilidades a su profesionalismo o carrerismo. En este sentido, en éticas profesionales no sólo hablamos de responsabilidad personal, sino de corresponsabilidades profesionales porque el ejercicio profesional no es una práctica individual sino que se realiza compartida, implicando a los afectados, a otros compañeros, a la institución que vertebramos e incluso a la propia familia. Una corresponsabilidad que hoy realizamos en un contexto económico y cultural nihilista donde el conocimiento aparece deshumanizado (sin tradiciones) y las identidades morales fragmentarias. Ante un nihilismo aparentemente inofensivo sólo nos cabe plantear una responsabilidad decididamente comunitaria y afirmativa. ${ }^{10}$

\section{2. ¿Por qué nos preocupa la ética de las profesiones?}

En la ética de las profesiones se ha producido una convergencia de inquietudes, que explica el interés que está despertando; éstas son las siguientes.

9 Sobreel concepto demediación, cfr. J. GiRó, (1998), "Lajusticiay la mediación, dos figuras diversas de la actividad comunicativa", en Educación Social 8, pp. 18-29; A. Domingo, (2002), "Claves éticas en la cultura de la mediación", en Introducción a la Ley de Mediación Familiar de la Comunidad Valenciana. Texto, esquemas y vocabulario, Valencia, Dirección General de la Familia.

10 Para una ampliación de estos planteamientos puede verse nuestro trabajo, (2002), Calidad Educativa y Justicia social, Madrid, PPC, caps. 3 y 4. 
a) La necesidad que tienen las instituciones universitarias de formar "buenos profesionales" y no sólo buenas personas o "profesionales buenos". Esta ética no puede reducirse a la bondad personal del profesional que, además de ser un buen técnico en su oficio, es una "buena persona". Para ser un buen profesional, la capacitación, cualificación o preparación técnica es necesaria, pero no suficiente. Ciertamente, las universidades tienen que estar preocupadas por la calidad de la moral personal de sus alumnos, pero la tarea de la ética profesional en la universidad no puede reducirse a la excelencia personal o individual de sus alumnos. En este caso, más que ética profesional se trataría de moralina personal que no afectaría al ejercicio profesional, a su capacitación técnica y a sus competencias. ${ }^{11}$

b) Hacer frente a la tendencia de quienes plantean la filosofía moral al margen de la razón práctica. En estos casos, la ética se presenta como arma arrojadiza de unos grupos contra otros, como si la única misión de la ética fuera prescribir sub specie aeternitatis, bien basándose en principios abstractos que administran los profesionales de la ética, bien siendo consecuencia lógica de presupuestos metafísicos. La ética de las profesiones aparece así como antiideología porque denuncia una razón práctica descarnada y ahistórica. Nace como consecuencia de la rehabilitación de la razón práctica que se ha producido con la hermenéutica contemporánea, a la que tanto han contribuido H. G. Gadamer y P. Ricoeur.

c) El interés práctico no puede confundirse con un interés pragmático. La recuperación de la razón práctica no es la simple recuperación de una razón instrumental donde la ética tiene que capacitar a los profesionales para el cálculo en la utilización de los medios técnicos. Para que este interés práctico sea un interés moral y pueda tener toda la fuerza de una antiideología, es importante plantear la profesión desde una ética de las actividades humanas, es decir, planteando el sentido de las prácticas profesionales en la constitución del carácter, las virtudes y la identidad moral de los profesionales.

d) Herramienta para evitar el autismo de las especialidades. La historia de las áreas y los departamentos universitarios es la historia de una especialización permanente que ha traído como consecuencia una pulverización y ensimismamiento de los investigadores en su propio

11 Cfr. A. HoRTAL, (2002), Ética general de las profesiones, Bilbao, Desclée de Brouwer. 
campo. En estos casos, la ética de las profesiones no sólo apela a la dimensión histórica y social del conocimiento, sino que plantea la pregunta por el modelo de ética ciudadana que necesitamos para la aldea global. ${ }^{12}$

e) La pregunta por el sentido y valor del trabajo como actividad humana identificante e identificadora. Una ética de las profesiones no puede quedar reducida a una ética de las profesiones que más historia tienen o que más tradición tienen como profesión. Nos preocupa la ética de quienes estudian profesiones tradicionales, pero no podemos estar al margen de nuevas profesiones que están apareciendo y que también precisan una organización de su reflexión moral. Eso sin contar la transformación que se ha producido de las profesiones convencionales donde actividades como la abogacía o la medicina ya no se realizan aisladamente sino que se realizan en redes y equipos de trabajo dentro de instituciones que condicionan la praxis. La ética de las profesiones puede romper planteamientos anquilosados de una ética basada en el simple aprendizaje de principios que debían aplicarse, en el análisis paradigmático de casos o en la resolución de conflictos que aparecen entre la legalidad social y la moralidad profesional. Los nuevos contextos económicos, políticos y sociales plantean de forma radicalmente nueva la relación entre identidad personal y vida profesional, entre la unidad narrativa del proyecto de vida y la diversidad de ocupaciones que constituyen un medio de vida. ${ }^{13}$

f) La privatización y psicologización de las actividades profesionales. La ética profesional no es la ética de un profesional privado sino que interviene directamente en la configuración de los valores sociales. La ética de las profesiones tiende un puente entre el árbol de la propia actividad y el bosque del modelo de sociedad que construimos. Una actividad profesional tiene una dimensión individual, pero también una dimensión pública: no es una pura relación mercantil o contractual, expresa el modo de realizar los valores, de hacer valiosa la vida.

12 Para los diferentes modelos de ciudadanía que presenta la ética contemporánea puede verse nuestro estudio, (2001), "Modelos de ciudadanía en la sociedad global", en Documentación social 125 , pp. $243-260$.

13 He realizado una aplicación de estos problemas al directivo de empresa en "Ética de la dirección", en A. Cortina, J. Conill, A. Domingo y D. García, (2003), Ética de la Empresa (sexta edición), Trotta, Madrid, cap. 4. 
Las decisiones profesionales cada vez son más decisiones colegiadas $\mathrm{y}$, por tanto, el alcance de las responsabilidades afecta a la legitimidad, plausibilidad y credibilidad de la propia profesión. La responsabilidad profesional no se limita al cumplimiento de la legalidad correspondiente, como si fuera un simple "ajuste de cuentas" para estar en paz, se trata de plantear la profesión y su sentido no sólo como fuentes de capital humano, sino como instrumentos privilegiados para la creación, mantenimiento y fortalecimiento del capital social ${ }^{14}$. Por eso, hoy sería más exacto hablar de corresponsabilidad profesional.

\section{La ética de las profesiones en la institución universitaria: cinco propuestas}

La ética de las profesiones desempeña un papel importante como eslabón entre la sociedad del conocimiento (consecuencia de la sociedad de la información) y la promoción de la justicia social. En este sentido, la ética de las profesiones es un puente privilegiado entre el capital humano y el capital social. Para que así sea, ¿cómo plantearla?

1. En primer lugar, manifestando ante la opinión pública que el conocimiento y su gestión no son moralmente inocentes. Cuando hoy hablamos de la sociedad de la información y, también, de la sociedad del conocimiento, tenemos la impresión de que términos como globalización y conocimiento son producto de factorías presididas por la inocencia moral. Así, gestionar la investigación, capacitación y formación de ciudadanos no es una tarea moralmente neutral o inocente. Por eso apelamos a la corresponsabilidad como principio clave de la ética de las profesiones.

Ahora bien, ¿por qué enfrentamos "nihilismo" con "responsabilidad"?, ¿qué papel desempeña un término con tanta fuerte carga filosófica como el de "nihilismo"? La razón es muy sencilla, las universidades pueden mantenerse indiferentes ante los desafíos culturales y morales, pueden organizarse como torres de marfil donde se produzca una alfabetización profesional pero no una alfabetización ciudadana de los profesionales. Las universidades pueden llegar a ser parásitos de la comunidad política y no plantearse su función pública. Es más, incluso pueden olvidarse de la estrecha relación

14 Para profundizar en el concepto de capital social y su relación con el capital humano, puede verse el último capítulo de mi libro, (2001), Ética. Todo lo que usted estudió y nunca debió olvidar (puesto al día), Madrid, Acento Editorial. 
que hay entre el sufrimiento humano y la generación del conocimiento. A esto nos referimos cuando hablamos de "nihilismo" como indiferencia de las universidades ante el sufrimiento, el empobrecimiento y el olvido de los programas de humanización. ${ }^{15}$

2. Manteniendo la dimensión magisterial y personal del conocimiento. La sociedad del conocimiento no puede ser una sociedad sin autoridades públicas, sin referencias morales, sin liderazgos que orienten a los pueblos. En una ética profesional, los alumnos deben saber que la información que se les transmite y la capacitación para la que se les prepara se realiza en continuidad con una tradición de saberes. Como profesionales están llamados a continuar y entregar a las próximas generaciones el testigo de un conocimiento científico-técnico que no es impersonal o ahistórico.

Esto no significa que la enseñanza tenga que ser partidista o ideologizada, significa descubrir que la universidad no es sólo un centro de transmisión de datos sino un espacio de creatividad para el descubrimiento de dotes y capacidades. La ética de las profesiones no sólo nos recuerda los límites del positivismo y del idealismo científico, sino la irresponsabilidad del escepticismo o del relativismo moral. La reflexión moral es un ámbito privilegiado para que se produzca una fusión de horizontes entre las disciplinas, las áreas y los departamentos. Son los propios desafíos de la sociedad los que llaman a la puerta de las universidades y las gentes no sólo demandan propuestas especializadas sino propuestas coherentes. La ética de las profesiones es un índice de calidad en las respuestas y, también, un índice de coherencia al evaluar la función social de la universidad. ${ }^{16}$

3 . Ejerciendo como instrumento privilegiado para que alumnos y profesores se planteen corresponsablemente la unidad narrativa de sus vidas. Además de profesionales adiestrados con un determinado capital humano, son ciudadanos implicados en el capital social de sus comunidades más próximas. La unidad narrativa no se consigue separando vida personal y vida

15 En este punto sería importante evaluar el valor que las universidades conceden al voluntariado. Un primer análisis de estas relaciones fue el que hice en, (2000), Ética y Voluntariado. Una solidaridad sin fronteras (segunda edición), Madrid, PPC.

16 Sobre el papel que desempeña en humanismo en general, y el humanismo cristiano en particular, en la estructuración ética de estas instituciones puede verse mi escrito "La deshumanización de la justicia social: la responsabilidad del humanismo cristiano en una democracia liberal", ponencia presentada en el III Encuentro de Fundaciones Democristianas, Barcelona, Enero 2005. Texto que aparecerá en la revista Diàlegs, 2005, en prensa. 
profesional, tampoco tejiendo de forma coherente las diferentes ocupaciones profesionales que una persona puede tener a lo largo de su vida. Se consigue cuando el profesional se plantea de forma radical su relación con el tiempo. Cuando decide ponerse frente a él y orienta su proyecto de vida personal, su vida familiar, su compromiso ciudadano y su carrera profesional. Cuando se plantean las diferencias entre la mentalidad del empleado y la mentalidad del profesional. Como ya hemos indicado en otros trabajos, el empleado se relaciona de una manera instrumental y externa con el tiempo, su hacer laboral y su identidad personal no sólo están claramente diferenciadas sino separadas. Por el contrario, el profesional se relaciona con el tiempo de forma interna, cuando hace algo no "emplea un tiempo" sino que comparte un tiempo "se emplea en el tiempo", al hacer (actividad) se hace a sí mismo (ser), su identidad personal no se entendería sin su identidad profesional.

Esto genera dos mentalidades muy diferentes con las que solemos diferenciar la mentalidad de empleado y la mentalidad de profesional. Simplificando un poco podemos verlas expuestas en esta tabla:

\begin{tabular}{|l|l|}
\hline \multicolumn{1}{|c|}{ Mentalidad de empleado } & \multicolumn{1}{c|}{ Mentalidad de profesional } \\
\hline $\begin{array}{l}\text { Relación externa (instrumental y } \\
\text { mecánica) con el tiempo }\end{array}$ & $\begin{array}{l}\text { Relación interna (constitutiva y } \\
\text { antropológica) con el tiempo }\end{array}$ \\
\hline Hacer algo & Hacerse haciendo \\
\hline Emplear el tiempo en algo & Emplearse en el tiempo \\
\hline Tener un trabajo & Ejercer una profesión \\
\hline $\begin{array}{l}\text { Separación proyecto personal y } \\
\text { proyecto laboral }\end{array}$ & $\begin{array}{l}\text { Coordinación proyecto personal y } \\
\text { proyecto profesional }\end{array}$ \\
\hline Ética del trabajo como necesidad & $\begin{array}{l}\text { El trabajo desde la libertad y } \\
\text { creatividad }\end{array}$ \\
\hline
\end{tabular}

4. Facilitando, desde la universidad, el entrenamiento en la capacidad de juicio y en la adquisición de una sabiduría práctica (fronesis) que no se consigue únicamente con el estudio de casos, con el conocimiento en la aplicación de principios, sino con el conocimiento crítico de la realidad social en su conjunto. Esta sabiduría práctica es cada día más necesaria para forjar el carácter, no sólo la personalidad profesional, el estilo de vida profesional o 
simplemente el talante profesional. Cuando hablamos de forjar el carácter no nos remitimos al ímpetu o la energía con la que los profesionales tienen que tomar decisiones arriesgadas, tampoco a la necesidad de resignarse a una determinada actividad. Nos referimos a la posibilidad de llegar a ser sujetos con personalidad propia, con proyecto de vida propio y a la necesidad de resistir ante un capitalismo flexible que está contribuyendo a la "corrosión del carácter" en la organización de la vida profesional. ${ }^{17}$

Esto no significa que las éticas profesionales condicionen a la institución universitaria para que ésta tenga que resistir y hacer frente necesariamente a determinado sistema económico. Significa que la institución universitaria no deja sin cuestionar el modelo de sociedad, así como las formas de entender el capitalismo, es decir, que la universidad es un espacio público donde se plantea con radicalidad la relación entre sistema económico, sistema socio-político y sistema moral. La intervención en el marco de un sistema económico capitalista no supone la aceptación de sus presupuestos antropológicos, éticos y sociales. En este sentido, la universidad es un espacio privilegiado para esclarecer estos presupuestos y plantear con profundidad la pregunta por el modelo de sociedad que sustenta un determinado sistema económico o político.

5. Finalmente, organizando las responsabilidades que la universidad tiene en la investigación, la docencia y la capacitación integral de sus miembros puede organizarse únicamente como si la universidad fuera un centro de servicios, es decir, un "club de formación profesional" o espacio social que desempeña una función instrumental de capacitar a los profesionales. Como centro de servicios, a la universidad se le pediría eficiencia, eficacia y economía; los individuos encuentran en ella unos servicios que le son de gran utilidad para su capacitación profesional. Ahora bien, la universidad también tiene la posibilidad de organizar sus responsabilidades como espacio de identificación, es decir, como referencia de valor, autoridad moral y fuente de significados en la construcción de la identidad personal y profesional.

La universidad puede tener en las éticas profesionales un instrumento para que los alumnos se planteen la autenticidad de su vocación, la coherencia de sus decisiones profesionales y la forma en la que administran corresponsablemente las oportunidades que su capacitación les ofrece. Las éticas profesionales pueden ser una ocasión importante para que pro-

17 Cf. R. SEnNetr, La corrosión del carácter, Anagrama, Barcelona, 2000. 
fesores y alumnos nos planteemos la unidad narrativa de nuestras prácticas profesionales, sociales y familiares. R. Sennett plantea el problema en los siguientes términos:

...cómo proteger las relaciones familiares para que no sucumban a los comportamientos a corto plazo, el modo de pensar inmediato y, básicamente, el débil grado de lealtad y compromiso que caracterizan el moderno lugar de trabajo. En lugar de los valores cambiantes de la nueva economía, la familia... debería valorar la obligación, la honradez, el compromiso, la finalidad.... Este conflicto entre familia y trabajo plantea algunas cuestiones sobre la experiencia de la vida adulta en sí. ¿Cómo pueden perseguirse objetivos a largo plazo en una sociedad a corto plazo? ¿ Cómo puede un ser humano desarrollar un relato de su identidad e historia vital en una sociedad compuesta de episodios y fragmentos? Las condiciones de la nueva economía se alimentan de una experiencia que va a la deriva en el tiempo, de un lugar a otro lugar, de un empleo a otro. Si pudiera establecer el dilema de Rico en términos más amplios, diría que el capitalismo de corto plazo amenaza con corroer su carácter, en especial aquellos aspectos del carácter que unen a los seres humanos entre sí y brindan a cada uno de ellos una sensación de un yo sostenible. ${ }^{18}$

\section{Tres modelos de responsabilidad profesional}

Voy a presentar tres modelos de profesionalización que puede ofrecer una institución universitaria. No tienen pretensión de completar todas y cada una de formas posibles en las que una universidad puede plantearse la organización de las éticas profesionales. Pretendo incentivar la reflexión sobre los diferentes modos de enfocar la responsabilidad profesional. Según cómo se entiende ésta en la institución universitaria, así se transmitirá a los alumnos y así se organizará el universo de significados con los que el profesional responderá a los desafíos éticos de su sociedad. Aunque planteo tres modelos, la ausencia de respuesta también representa un modelo que podríamos llamar naturalista o nihilista, por eso quiero dejar claro que el nihilismo es una opción a la que se están apuntando las universidades cuando no se plantean explícitamente la responsabilidad profesional.

Sería una profesionalización que podríamos llamar profesionalización ironista utilizando el término "ironía" en el sentido que R. Rorty le da al término. A su juicio hay dos grandes actitudes ante la vida, por un lado la de quienes se la toman en serio y, por otro, la de quienes se sitúan irónicamente. Una profesionalización ironista sería, a nuestro juicio, una estrategia de profesionalización irresponsable porque se desentendería de la dimensión

18 Cf. R. SEnNETt, op. cit., p. 25. 
moral del conocimiento, de los procesos de capacitación y, sobre todo, del modelo de profesional al que la universidad se propone formar. Utilizando las reflexiones de Rorty, Sennett comenta lo siguiente:

\begin{abstract}
...la ironía es un estado mental en el que la gente nunca es totalmente capaz de tomarse a sí mismo en serio porque siempre es consciente de que los términos en los que se describe están sujetos a cambio, siempre es consciente de la contingencia y la fragilidad de su vocabulario...es la consecuencia lógica de vivir un tiempo flexible, sin criterios de autoridad o responsabilidad... la ironía tampoco estimula a la gente a desafiar el poder... ${ }^{19}$
\end{abstract}

\title{
4.1. Profesionalización mecanicista
}

El primer modelo puede ser descrito como profesionalización mecanicista. Lo llamo así porque la universidad se adapta y ajusta a los mecanismos del mercado laboral en su planteamiento formativo. Ante los procesos de modernización se situaría en términos económicos y utilitaristas. Una universidad al servicio de la sociedad sería aquella que proporciona sólo los profesionales que necesitan los procesos de racionalización social y crecimiento económico. El fin de una ética profesional sería el entrenamiento para el cálculo basado en el principio de la triple $E$ : economía, eficiencia y eficacia.

Esta opción supone una determinada forma de organizar el conocimiento caracterizada por la separación, un profesional es un experto, un especialista, una persona con determinadas destrezas o habilidades técnicas. La universidad no se tendría por qué encargar de otro tipo de habilidades que tuvieran que ver con la responsabilidad social, la forja del carácter, la voluntad de servicio o el sentido de la justicia. Como mucho, mantendría un servicio de atención psicopedagógica porque estos problemas son problemas de desajuste individual, de desadaptación social o de algún trastorno psicológico. Éstos son asuntos que o bien son privados y debe ser resueltos por el propio estudiante en su vida privada o por el conocimiento de la legislación. La identidad profesional se limitaría a una identidad técnica, sin plantear para nada dimensiones antropológicas, sociales o culturales. La ética profesional facilita la adaptación a contextos de individualismo y atomismo moral. Esta adaptación pragmática es la única que garantiza el éxito y triunfo en el estatus socio-económico.

Este planteamiento mostraría que la universidad no se plantea los fines

19 Cf. R. SenNett, op. cit., p. 122. 
de la ciencia y la técnica sino que se adapta a ella, cree en ella y, por consiguiente, transmite involuntariamente la utopía científico-tecnológica de un progreso indefinido. La formación para la responsabilidad se limitaría al entrenamiento para el cumplimiento de la legalidad y para el ajuste o adaptación psico-orgánica del sujeto. La solidaridad se entendería como filantropía, como producto de la liberalidad del profesional que graciosamente colabora con causas justas. Habría una relación directa entre liberalidad y solidaridad sin transitar por el terreno de otra justicia que no sea la estrictamente legal.

\subsection{Profesionalidad civilizadora}

Un segundo modelo puede ser descrito como profesionalidad civilizadora, llamado así porque la universidad no sólo se plantea responder a la modernización económica sino a una modernización cívica. La ética profesional tiene que capacitar para profesionales que no sólo deben hacer cálculo económico sino cálculo social, es decir, que además de estar preocupados por el crecimiento económico o el cumplimiento de las leyes han de estar preocupados por el desarrollo social y el bienestar de la sociedad.

La ética de las profesiones supondría también una organización del conocimiento donde los saberes se subordinarían a la planificación social, al desarrollo de instituciones de bienestar. Seguiría existiendo una capacitación profesional con una fuerte dimensión pública y con un arrinconamiento de la dimensión personal o privada. Ahora bien, esta formación sería social, política y cultural. No se formaría para un individualismo o atomismo social en sentido estricto sino que se formaría para un "individualismo responsable". A diferencia del modelo anterior que capacitaría para un individualismo irresponsable donde el éxito se plantea a corto plazo, en éste interviene la preocupación por los demás, la conciencia de dependencia y cohesión social. También la consideración de las consecuencias en términos de "largo plazo" y las decisiones no se tomarían únicamente en términos de inmediatez. ${ }^{20}$

La responsabilidad profesional sería una corresponsabilidad pública que ya no se limita a la responsabilidad legal mínima sino que cuenta con aspectos medio ambientales, laborales, culturales y familiares. La solidaridad se aproximaría a la justicia distributiva, transitaría su camino. Ahora no se

20 Cf. G. Lipovetsky, (1986), La era del vacío, Barcelona, Anagrama; ID., (1994), El crepúsculo del deber, Barcelona, Anagrama. 
trataría de una solidaridad filantrópica sino la solidaridad del mecenas o del profesional comprometido que se siente en la obligación de "devolver a la sociedad" lo que ésta le proporcionó. Muy próximo a lo que hoy entendemos por Responsabilidad Social Corporativa.

\subsection{Profesionalización identificante}

Un tercero y último modelo puede ser descrito como el de una profesionalización identificante, sería aquella donde el profesional tiene ocasión de situar su especialización y su capacitación en un conjunto amplio de valores y significados. Ni la economía, ni la sociología son fuentes últimas de sentido. Los valores y su organización serían determinantes en el modo de entender la ética profesional. Los profesionales no sólo son expertos, tampoco sólo son buenos ciudadanos, aspiran a ser personas que tienen un proyecto de vida que comparten, aspiran a unas sociedades abiertas donde pueden generar valor desarrollando su capacidad de innovación, creatividad y servicio. Son profesionales donde los valores sociales no se analizan únicamente en clave estatal o mercantil sino en clave creativa donde desempeñan un papel importante categorías morales como las de interioridad, singularidad o subjetividad de la sociedad. También son ciudadanos generadores de intangibles, administradores de confianza social y de credibilidad. La ética profesional facilita la legitimación no sólo económica o jurídica de una profesión sino su credibilidad social, su sentido histórico y su contribución al crecimiento personal en contextos comunitarios de bien común.

En la organización del conocimiento, la capacitación profesional no dejaría de lado el valor de las tradiciones, las religiones y las fuentes de sentido. Esto no significa su aceptación incondicional, sino el entrenamiento de los profesionales para que puedan comprender ámbitos de sentido que afectan al ensanchamiento o profundización de la interioridad, a la clarificación de los sentimientos o emociones y, sobre todo, ámbitos simbólicos inexplicables con el puro cálculo de la razón instrumental. El conocimiento se organizaría no sólo en términos de comunicación social, sino en términos de comunicación humana, es decir, implicando transformaciones y cambios internos de las personas. La palabra no sólo tiene una dimensión externa de enlace, relación y puente, sino una dimensión interna de diálogo interior, crecimiento personal y maduración en la búsqueda de la autenticidad. Estaríamos ante el proyecto de ética profesional del personalismo comunitario que integra dimensión cognitiva y dimensión simbólica de la inteligencia humana. 
La corresponsabilidad sólo se podría entender como solidaria. La corresponsabilidad profesional no sólo es la responsabilidad técnica o social, también la religación personal, la coimplicación personal en un modelo de sociedad, el compromiso personal en la utilización del conocimiento y las habilidades. La solidaridad profesional no sólo será filantropía o mecenazgo, sino preocupación por la justicia social y determinación en la consecución del bien común. ${ }^{21}$

\section{Conclusiones}

Como hemos visto, el debate sobre la ética de las profesiones es también un debate sobre el sentido y valor de la formación que estamos impartiendo en nuestras universidades. No podemos hablar de la responsabilidad profesional en un oficio determinado, en una carrera o en un itinerario universitario si antes no nos hemos planteado el modelo de corresponsabilidad profesional con el que estamos organizando la adaptación de nuestros centros al espacio europeo de educación superior o cualesquiera otro espacio educativo. La Ética de las profesiones desempeña una función mediadora porque tiende un puente entre las tradiciones de conocimiento y las exigencias de la sociedad, entre las diferentes especialidades y la necesidad de una mínima cultura moral común, entre los intereses pragmáticos de los diferentes grupos sociales y la creación de una ciudadanía crítica, entre la utilidad del trabajo como medio de vida y la actividad profesional como fuente de identidad.

Esta función mediadora es especialmente importante como eslabón perdido (y encontrado) entre la sociedad del conocimiento y la promoción de una cultura de la solidaridad. Por eso una ética de las profesiones no se limita a recordar el simple catálogo de deberes de una profesión o la necesidad de armonizar los intereses personales con el bien común. La Ética de las profesiones tiene que ser mucho más ambiciosa. Las instituciones que gestionan el conocimiento han de enfrentarse al nihilismo cultural mediante una nueva ética de la responsabilidad que no puede dar la espalda a las convicciones porque éstas no son simples principios morales arbitrarios sino disposiciones éticas que se adquieren a través de la información, la formación, la virtud, el diálogo y la voluntad de buscar coherencia y autenticidad en los proyectos de vida.

21 Cfr. A. Domingo, (2002), Educar para una ciudadanía responsable, Madrid, CCS-ICCE. 
En definitiva, estos tres modelos de responsabilidad profesional no se limitan únicamente a desarrollar diferentes formas de entender la ética de las profesiones. Nos plantean también la forma de entender la organización del conocimiento, los fines de la educación universitaria en una sociedad liberal y la inquietante pregunta por "un ser humano educado". Como vemos, la cuestión que planteábamos al principio no ha desaparecido pero esperemos que ahora no suponga, como afirmaba Bloom, una amenaza para la paz. 